\title{
State concentration exponent as a measure of quickness in Kauffman-type networks
}

\author{
Shun-ichi Amari, ${ }^{1}$ Hiroyasu Ando, ${ }^{1}$ Taro Toyoizumi, ${ }^{1}$ and Naoki Masuda ${ }^{2,3}$ \\ ${ }^{1}$ RIKEN Brain Science Institute, Hirosawa 2-1, Wako, Saitama 351-0198, Japan \\ ${ }^{2}$ Department of Mathematical Informatics, The University of Tokyo, 7-3-1 Hongo, Bunkyo, Tokyo 113-8656, Japan \\ ${ }^{3}$ PRESTO, Japan Science and Technology Agency, 4-1-8 Honcho, Kawaguchi, Saitama 332-0012, Japan \\ (Received 29 February 2012; revised manuscript received 24 October 2012; published 21 February 2013)
}

\begin{abstract}
We study the dynamics of randomly connected networks composed of binary Boolean elements and those composed of binary majority vote elements. We elucidate their differences in both sparsely and densely connected cases. The quickness of large network dynamics is usually quantified by the length of transient paths, an analytically intractable measure. For discrete-time dynamics of networks of binary elements, we address this dilemma with an alternative unified framework by using a concept termed state concentration, defined as the exponent of the average number of $t$-step ancestors in state transition graphs. The state transition graph is defined by nodes corresponding to network states and directed links corresponding to transitions. Using this exponent, we interrogate the dynamics of random Boolean and majority vote networks. We find that extremely sparse Boolean networks and majority vote networks with arbitrary density achieve quickness, owing in part to long-tailed in-degree distributions. As a corollary, only relatively dense majority vote networks can achieve both quickness and robustness.
\end{abstract}

DOI: 10.1103/PhysRevE.87.022814

PACS number(s): 89.75.Hc, 87.18.Sn, 05.45.-a

\section{INTRODUCTION}

Networks of binary elements are useful tools for investigating a plethora of dynamical behavior and information processing in biological and social systems. For example, various models of associative memory are used to study neural information processing [1-3]. Random Boolean networks, also known as Kauffman nets, show rich dynamics and are used to model gene regulation [4-7]. Random majority vote networks are often used to understand mechanisms for ordering in neural information processing [3,8-10], gene regulation [7], and collective opinion formation in social systems [11]. We study the dynamics of such networks by using a simple generative model of randomly connected Boolean and majority vote elements in the cases of sparse and dense connectivity.

Properties desirable for the dynamics of networks of such binary units include robustness and quickness. A system is defined to be robust when the flipping of a small number of units' states does not eventually alter the behavior of the entire network. For random Boolean networks, the robustness has been quantified in the context of damage spreading in cellular automata [12-15].

Dynamics is usually called quick if an orbit starting from an arbitrary state reaches the corresponding attractor within a small number of steps on average, i.e., with a short transient length of the dynamics. However, even the average transient length, which apparently seems to be the most basic quantity to characterize the statistics of the transient length, may be difficult to evaluate because the transient length of the random Boolean networks seems analytically intractable and it obeys long-tailed distributions [16]. Therefore, in this paper, we theoretically study the quickness of dynamics by use of

Published by the American Physical Society under the terms of the Creative Commons Attribution 3.0 License. Further distribution of this work must maintain attribution to the author(s) and the published article's title, journal citation, and DOI. the concept of state concentration instead of examining the transient length. To this end, we extend the previous statistical dynamical framework [8-10]. In particular, the exponent of concentration, which we introduce later, is an analytically tractable quantity for measuring the quickness of dynamics in random Boolean and majority vote networks. Using this exponent, we investigate the compatibility of the robustness and quickness in these two types of networks in two cases of connectivity, i.e., sparse and dense connectivity.

For this purpose, we distinguish densely connected Boolean networks (DBNs), sparsely connected Boolean networks (SBNs), densely connected majority vote networks (DMNs), and sparsely connected majority vote networks (SMNs). We elucidate the differences between the four dynamics. In particular, we show that strong state concentration, accompanied by a power law type of in-degree distribution with an exponential cutoff, occurs in the majority vote networks (DMNs and SMNs) but not for the Boolean networks except for extremely sparse cases. Then, we argue that DMNs are the only type among the four types of network that realizes both robustness and quickness.

\section{MODEL}

Let us consider a network of $n$ binary units. We define the discrete-time dynamics of the network by

$$
x_{i}(t+1)=f_{i}\left(x_{1}(t), \ldots, x_{n}(t)\right) \quad(1 \leqslant i \leqslant n),
$$

where $x_{i}(t) \in\{1,-1\}$ is the binary state of the $i$ th unit at time $t$. For a random Boolean network, each $f_{i}$ is randomly and independently chosen from the $2^{2^{n}}$ Boolean functions on the $n$ units. For a majority vote network,

$$
f_{i}\left(x_{1}, \ldots, x_{n}\right)=\operatorname{sgn}\left(\sum_{j=1}^{n} w_{i j} x_{j}\right) \quad(1 \leqslant i \leqslant n),
$$

where sgn indicates the sign function. We consider an ensemble of randomly generated majority vote networks where $w_{i j}$ 
are independently and identically distributed Gaussian random variables. In general, a constant or random threshold could be included in the above dynamical expression, which we omit here for simplicity. If the value of $f_{i}\left(x_{1}, \ldots, x_{n}\right)$ depends only on randomly chosen $K$ units for each $i$, the model is called the $K$-sparse network [5,6]. DBNs and DMNs correspond to $K \propto n$, and SBNs and SMNs correspond to $K \ll n$. We study typical dynamical behavior of random DBNs, SBNs, DMNs, and SMNs.

The number of possible functions generated by a single unit in the four types of network is compared as follows. The number of all Boolean functions is equal to $2^{2^{n}}$, growing doubly exponentially with $n$. This is equal to the variety of the random mapping on $n$ units. The number of functions generated by a single unit in DBNs is large for large $K$ and equal to $2^{2^{n}}$ when $K=n$. In contrast, SBNs, DMNs, and SMNs are limited in terms of the number of possible functions. The number of all majority vote units is asymptotically equal to $2^{n^{2} / 2}$; the growth rate is only exponential. For the sparse Boolean and majority vote networks, the number of functions generated by a single unit is equal to $2^{K \log _{2} n}$, growing only algebraically with $n$. The differences in the variety of functions in the four cases may result in different dynamical behaviors of the networks, as we will analyze in the following.

\section{DISTANCE LAW IN STATE TRANSITIONS}

The network state at time $t$ is given in vector form as

$$
\boldsymbol{x}(t)=\left(x_{1}(t), \ldots, x_{n}(t)\right) .
$$

Let $X=\{\boldsymbol{x}\}$ be the set of the $N \equiv 2^{n}$ states. Given a network, the state transition is a mapping from $X$ to itself. We write it briefly as $\boldsymbol{x}(t+1)=f \boldsymbol{x}(t)$.

The dynamics of the distance between two state trajectories has been studied to characterize dynamics in these networks. We define the normalized Hamming distance between two states $\boldsymbol{x}$ and $\boldsymbol{y}$ in $X$ by

$$
D(\boldsymbol{x}, \boldsymbol{y})=\frac{1}{2 n} \sum_{i=1}^{n}\left|x_{i}-y_{i}\right| .
$$

It should be noted that the distance is restricted to the range $0 \leqslant D(\boldsymbol{x}, \boldsymbol{y}) \leqslant 1$. We let $d=D(\boldsymbol{x}, \boldsymbol{y})$ and $d^{\prime}=D(f \boldsymbol{x}, f \boldsymbol{y})$. The mapping from $d$ to $d^{\prime}$ is a random variable and depends on $\boldsymbol{x}$ and $\boldsymbol{y}$ because $w_{i j}$ is a random variable and many pairs of $\boldsymbol{x}$ and $\boldsymbol{y}$ realize $d=D(\boldsymbol{x}, \boldsymbol{y})$. However, we can prove that $d^{\prime}=\varphi(d)$ for a function $\varphi(d)$ for any $\boldsymbol{x}$ and $\boldsymbol{y}$ almost always as $n \rightarrow \infty$. We call $\varphi(d)$ the distance law. For DBNs, $\varphi(d)=0$ $(d=0)$ and $\varphi(d)=1 / 2(d \neq 0)$. For SBNs [17],

$$
\varphi(d)=(1 / 2)\left[1-(1-d)^{K}\right] .
$$

For DMNs [8-10],

$$
\varphi(d)=(2 / \pi) \sin ^{-1} \sqrt{d} .
$$

For SMNs [18],

$$
\varphi(d)=\sum_{j=0}^{K} g_{K, j}\left(\begin{array}{c}
K \\
j
\end{array}\right) d^{j}(1-d)^{K-j},
$$

where $\left(\begin{array}{c}K \\ j\end{array}\right)$ is the binomial coefficient and

$$
g_{K, j}=(2 / \pi) \sin ^{-1} \sqrt{j / K} .
$$

The dynamics of the distance under the annealed approximation is given by $d_{t+1}=\varphi\left(d_{t}\right)$ [8,17-19]. For all four types of network, $\varphi(0)=0$. For DBNs, $\varphi(d)$ is discontinuous at $d=0$, and the dynamics is essentially the same as that of a random mapping on the $N$ states. Therefore, various properties of the dynamics such as the number of attractors, transient length, and cycle period are well characterized $[5,6,8]$. For SBNs, DMNs, and SMNs, $\varphi(d)$ is continuous at $d=0$. It is known that $d=0$ is a stable fixed point of mapping $\varphi$ [i.e., $0<\varphi^{\prime}(0)<1$ ] only for SBNs and SMNs with $K=1$ or 2 . Otherwise, $d_{t}$ converges to a positive value $\bar{d}$ satisfying $\bar{d}=\varphi(\bar{d})$. The convergence of the distance is usually fast and happens after $\sim 10$ steps except for DBNs where one step is enough for the distance to converge.

\section{EXPONENT OF THE STATE CONCENTRATION}

The average transient length before the orbit enters the attractor is analytically intractable. In addition, it may not be a good measure of the quickness of the dynamics due to the long-tailed nature of its distribution [16]. Therefore, we introduce an alternative order parameter called the exponent of the state concentration. We use the so-called state transition graph $[5,6,20]$ defined as follows. A map $f$, either Boolean or majority vote, induces a graph on $N$ nodes. Each state $\boldsymbol{x} \in X$ defines a node and has exactly one outgoing link directed to node $f \boldsymbol{x}$.

Suppose that each of the $N=2^{n}$ nodes (i.e., states) has a token at $t=0$. For each $t(\geqslant 0)$, an application of $f$ moves all the tokens at each node $\boldsymbol{x}$ to node $f \boldsymbol{x}$. Repeated applications of the mapping $f$ elicit concentrations of tokens at specific nodes. We denote by $f^{-t} \boldsymbol{x}$ the set of nodes whose tokens move to $\boldsymbol{x}$ after $t$ steps and by $\left|f^{-t} \boldsymbol{x}\right|$ the number of tokens at node $\boldsymbol{x}$ after $t$ steps. Tokens are initially equally distributed, i.e., $\left|f^{0} \boldsymbol{x}\right|=1$ for each $\boldsymbol{x}$, and the total number of tokens is conserved throughout the dynamics, i.e.,

$$
\sum_{\boldsymbol{x} \in X}\left|f^{-t} \boldsymbol{x}\right|=N
$$

for $t \geqslant 0$.

The in-degree of node $x$ in the state transition graph is equal to $\left|f^{-1} \boldsymbol{x}\right|$. The nodes with $f^{-1} \boldsymbol{x}=\emptyset$, where $\emptyset$ is the empty set, do not have parent nodes. The set of such nodes is called the Garden of Eden [5] and denoted by $E_{1}$. The nodes $\boldsymbol{x} \in E_{1}$ appear only as initial states. Only the nodes $x \in X-E_{1}$ retain tokens at $t=1$. In general, we define $E_{t} \equiv\left\{\boldsymbol{x} \mid f^{-t} \boldsymbol{x}=\emptyset\right\}$, i.e., the set of nodes that do not have tokens at time step $t$. There exists integer $T$ such that

$$
\phi \subset E_{1} \subset E_{2} \subset \cdots \subset E_{T}=E_{T+1}=\cdots \equiv E^{*},
$$

where $T$ is the longest transient period and $E^{*}$ is the set of the transient states. The set of the attractors is given by $A=$ $X-E^{*}$ (Fig. 1).

To quantify the state concentration, we consider the number of tokens that a token at $\boldsymbol{x}(0)$ and $t=0$ meets after we apply $f$. We write the relationship $f \boldsymbol{x}(0)=\boldsymbol{x}(1)$ succinctly 


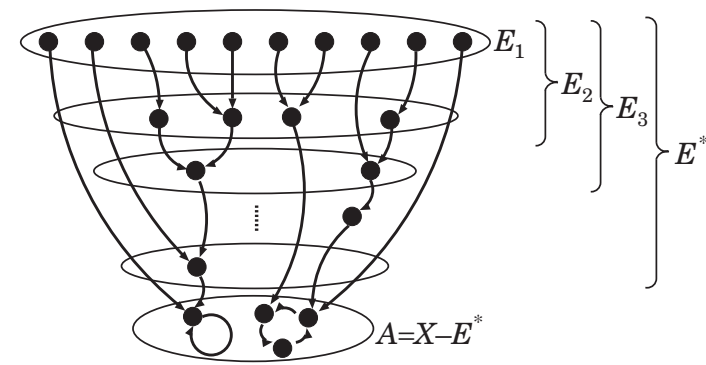

FIG. 1. Schematic of the dynamics of state concentration. $E_{1}$ indicates the Garden of Eden, $E_{2}$ indicates the nodes that do not have grandparents, and $A$ indicates the set of attractors.

as $\boldsymbol{x}(0) \rightarrow \boldsymbol{x}(1)$. Let $\bar{S}_{1}$ be the conditional expectation $E\left[\left|f^{-1} \boldsymbol{x}(1)\right| \mid \boldsymbol{x}(0) \rightarrow \boldsymbol{x}(1)\right]$ of the in-degree of node $\boldsymbol{x}(1)$ [i.e., the number of parents of $x(1)$ ] given that $x(0) \rightarrow x(1)$ and that $\boldsymbol{x}(0)$ is selected with equal probability (i.e., $1 / N)$. In general, we denote by $\bar{S}_{t}$ the expected number of $t$-fold ancestor nodes of a node $\boldsymbol{x}(t)$ conditioned by a state transition path ending at $\boldsymbol{x}(t)$ through which a token has traveled, or equivalently, conditioned by the uniformly distributed initial token $\boldsymbol{x}(0)$, i.e.,

$$
\bar{S}_{t} \equiv E\left[\left|f^{-t} \boldsymbol{x}(t)\right| \mid \boldsymbol{x}(0) \rightarrow \cdots \rightarrow \boldsymbol{x}(t-1) \rightarrow \boldsymbol{x}(t)\right] .
$$

Obviously $\bar{S}_{0}=1$ and the sequence $\left\{\bar{S}_{t}\right\}$ is monotonically nondecreasing in $t$. If

$$
\bar{S}_{t}=e^{c_{t} n}, \quad c_{t}>0,
$$

holds true for large $n$, the tokens are exponentially concentrated on nodes having at least a $t$-fold ancestor node. We refer to

$$
c_{t}=\lim _{n \rightarrow \infty} \frac{\ln \bar{S}_{t}}{n}
$$

as the $t$-step exponent of the concentration. The exponent $c_{t}$ quantifies the degree of state concentration and is a measure of quickness. It should be noted that we do not need to explicitly evaluate the statistics of the transient to calculate $c_{t}$.

The stochastic symmetry of units makes the calculation of $\bar{S}_{t}$ tractable. To explain the symmetry, we consider the majority vote network; similar arguments hold true for the Boolean network. Because the weights $w_{i j}$ are independently and identically distributed, the probability distribution of the mapping $f$ is invariant under permutation of $x_{1}, \ldots, x_{n}$, which are passed as the arguments to $f_{i}(1 \leqslant i \leqslant n)$. In addition, the probability is invariant under flip of the sign of each $x_{i}$. Therefore, the following gauge invariance holds. First, the probability distribution of $\boldsymbol{x}(t)$ is invariant under permutation of the unit indices. Second, the probability distribution is invariant under a sign flip of any $x_{i}(t)$. Because any state is mapped in a single step to a given $\boldsymbol{x}$ by permutation and sign flip, all the states are stochastically equivalent. Therefore, $\operatorname{Prob}\left\{\left|f^{-1} \boldsymbol{x}\right|=k\right\}$, for example, is the same for all $\boldsymbol{x}$, and $\operatorname{Prob}\{\boldsymbol{x}(0) \rightarrow \boldsymbol{x}(1)\}=1 / N$ for any $\boldsymbol{x}(0)$ and $\boldsymbol{x}(1)$.

We define a conditional probability distribution

$$
r_{k}=\operatorname{Prob}\left\{\left|f^{-1} \boldsymbol{x}(1)\right|=k \mid \boldsymbol{x}(0) \rightarrow \boldsymbol{x}(1)\right\},
$$

which is the in-degree distribution of node $x(1)$ conditioned by $\boldsymbol{x}(0) \rightarrow \boldsymbol{x}(1)$. The symmetry guarantees that $r_{k}$ is independent

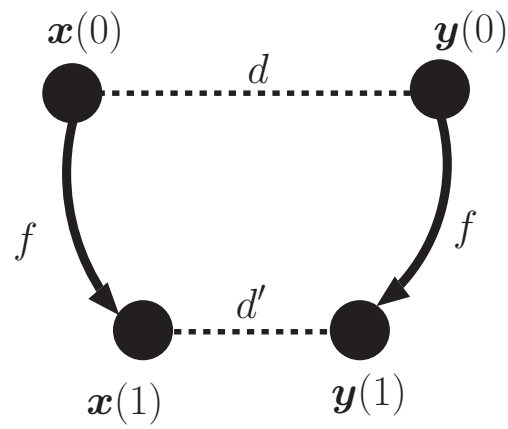

FIG. 2. Schematic of one-step dynamics of the distance.

of $\boldsymbol{x}(0)$ and $\boldsymbol{x}(1)$. Let us compute

$$
\bar{S}_{1}=\sum_{k=1}^{N} k r_{k} .
$$

We denote by $\boldsymbol{y}(0)$ a node such that $D(\boldsymbol{x}(0), \boldsymbol{y}(0))=d$ for a given $\boldsymbol{x}(0)$. The number of nodes with distance $d$ away from $\boldsymbol{x}(0)$ is given by

$$
\left(\begin{array}{c}
n \\
n d
\end{array}\right) \approx e^{n H(d)},
$$

where

$$
H(d) \equiv-d \ln d-(1-d) \ln (1-d)
$$

is the entropy. Because $\varphi(d)$ is the probability that the $i$ th components of $f \boldsymbol{x}(0)$ and $f \boldsymbol{y}(0)$ differ for any $i$, the probability that $D(f \boldsymbol{x}(0), f \boldsymbol{y}(0))=d^{\prime}$ (see Fig. 2 for a schematic illustration of this situation) is given by the binomial distribution as follows:

$$
\psi\left(d^{\prime} \mid d\right) \equiv\left(\begin{array}{c}
n \\
n d^{\prime}
\end{array}\right) \varphi(d)^{n d^{\prime}}[1-\varphi(d)]^{n\left(1-d^{\prime}\right)} .
$$

In particular,

$$
\begin{aligned}
\psi(0 \mid d) & =[1-\varphi(d)]^{n} \\
& =\operatorname{Prob}\{f \boldsymbol{y}(0)=\boldsymbol{x}(1) \mid \boldsymbol{x}(0) \rightarrow \boldsymbol{x}(1)\} .
\end{aligned}
$$

By using the saddle-point approximation, we obtain

$$
\begin{aligned}
\bar{S}_{1} & =\sum_{n d=0}^{n}\left(\begin{array}{c}
n \\
n d
\end{array}\right) \psi(0 \mid d) \\
& \approx \int \exp n\{H(z)+\ln [1-\varphi(z)]\} d z \propto e^{n c_{1}},
\end{aligned}
$$

where

$$
c_{1}=H\left(d^{*}\right)+\ln \left[1-\varphi\left(d^{*}\right)\right]
$$

and

$$
d^{*}=\underset{d}{\arg \max }\{H(d)+\ln [1-\varphi(d)]\} .
$$

To evaluate $c_{t}$ in general, we consider a $t$-step state transition path $X_{t}=\left\{\boldsymbol{x}(0) \rightarrow \boldsymbol{x}(1) \rightarrow \cdots \rightarrow \boldsymbol{x}(t)=\boldsymbol{x}^{*}\right\}$ ending at $x^{*}$ and calculate the conditional probability that another path $Y_{t}=\{\boldsymbol{y}(0) \rightarrow \cdots \rightarrow \boldsymbol{y}(t)\}$ ends at the same $\boldsymbol{x}^{*} . \bar{S}_{t}$ is the expectation of the number of such $t$-step paths. Let us denote the distance $D\left(\boldsymbol{x}\left(t^{\prime}\right), \boldsymbol{y}\left(t^{\prime}\right)\right)$ by $d_{t^{\prime}}$, where $0 \leqslant t^{\prime} \leqslant t$ and $d_{t}=0$. Then, under the Markov assumption, the probability of path $Y_{t}$ 


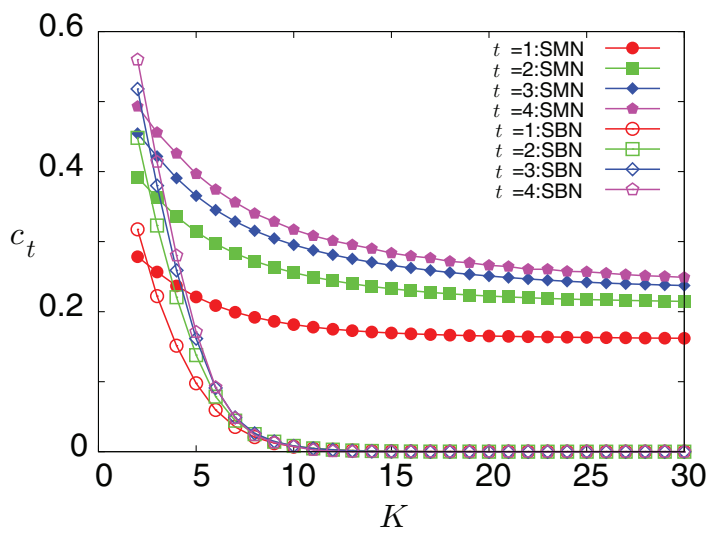

FIG. 3. (Color online) $c_{1}, c_{2}, c_{3}$, and $c_{4}$ for SMNs and SBNs.

conditioned by path $X_{t}$ is represented in terms of the distances of the two sequences, i.e., $d_{t^{\prime}}, 0 \leqslant t^{\prime} \leqslant t$, by

$$
\operatorname{Prob}\left\{Y_{t} \mid X_{t}\right\}=\prod_{t^{\prime}=1}^{t^{\prime}=t} \psi\left(d_{t^{\prime}} \mid d_{t^{\prime}-1}\right) .
$$

The Markov assumption is valid for large $n$ and a finite $t$. Because there are $\left(\begin{array}{c}n \\ n d_{0}\end{array}\right)$ states $\boldsymbol{y}(0)$ possessing distance $d_{0}$ from $\boldsymbol{x}(0)$, the expected number of paths is given by the integration of $\left(\begin{array}{c}n \\ n d_{0}\end{array}\right)$ Prob $\left\{Y_{t} \mid X_{t}\right\}$ over all the possible distance sequences $\left\{d_{0}, \ldots, d_{t-1}, d_{t}=0\right\}$. By using the saddle-point approximation, we have

$$
\bar{S}_{t}=\exp \left[n H\left(d_{0}^{*}\right)+\sum_{t^{\prime}=1}^{t} \ln \psi\left(d_{t^{\prime}}^{*} \mid d_{t^{\prime}-1}^{*}\right)\right]
$$

for large $n$, where $d_{t^{\prime}}^{*}$ are the maximizers of the integrand in the path integration. Equation (24) implies

$$
\begin{aligned}
c_{t}= & \sum_{t^{\prime}=1}^{t}\left\{H\left(d_{t^{\prime}-1}^{*}\right)+d_{t^{\prime}}^{*} \ln \varphi\left(d_{t^{\prime}-1}^{*}\right)\right. \\
& \left.+\left(1-d_{t^{\prime}}^{*}\right) \ln \left[1-\varphi\left(d_{t^{\prime}-1}^{*}\right)\right]\right\} .
\end{aligned}
$$

For example, for $t=2$, we obtain

$$
\begin{aligned}
c_{2}= & H\left(d_{0}^{*}\right)+H\left(d_{1}^{*}\right)+\ln \left[1-\varphi\left(d_{1}^{*}\right)\right]+d_{1}^{*} \ln \varphi\left(d_{0}^{*}\right) \\
& +\left(1-d_{1}^{*}\right) \ln \left[1-\varphi\left(d_{0}^{*}\right)\right],
\end{aligned}
$$

where

$$
\begin{aligned}
\left\{d_{0}^{*}, d_{1}^{*}\right\}= & \arg \max _{d_{0}, d_{1}}\left\{H\left(d_{0}\right)+H\left(d_{1}\right)+\ln \left[1-\varphi\left(d_{1}\right)\right]\right. \\
& \left.+d_{1} \ln \varphi\left(d_{0}\right)+\left(1-d_{1}\right) \ln \left[1-\varphi\left(d_{0}\right)\right]\right\} .
\end{aligned}
$$

On the basis of the expression of $\varphi$ for SBNs and SMNs shown before, the dependence of $c_{1}, c_{2}, c_{3}$, and $c_{4}$ on $K$ is plotted in Fig. 3. For SBNs, $c_{t}(1 \leqslant t \leqslant 4)$ converges to 0 quickly as $K$ increases. For DBNs, which is the case for $K=n$, we trivially obtain $c_{t}=0$ at least for small $t$ because $f$ is equivalent to the random mapping. Figure 3 indicates that state concentration occurs only for very small $K$ in the random Boolean network. In contrast, state concentration occurs even for large $K$ in majority vote networks. In particular, for DMNs with $K \rightarrow n$, we obtain $c_{1} \approx 0.157$ [10]. Figure 3 also indicates that the state concentration quickly proceeds as $t$ increases, except in DBNs.
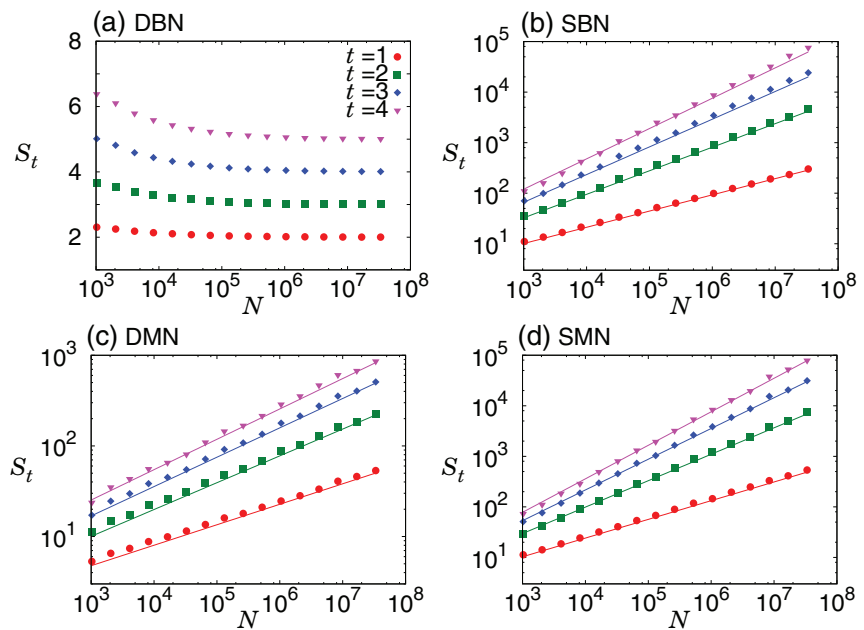

FIG. 4. (Color online) $\bar{S}_{t}$ exponentially increases with $n$ except in DBNs, (a) DBNs, (b) SBNs with $K=3$, (c) DMNs, and (d) SMNs with $K=3$. The lines in (b), (c), and (d) indicate the theoretical estimates (see Table I). Each point in the figure represents the average of $\bar{S}_{t}$ over $10^{3}$ realizations of the network.

We verified Eq. (13) by comparing $\bar{S}_{t}$ obtained from direct numerical simulations [i.e., Eq. (11)] and $c_{t}$ generally given by Eq. (25). The results shown in Fig. 4 indicate that the theory (lines) seems to agree with numerical results at least for large $n$; although the largest $n$ value shown in the figure is only $n=25$. Therefore, the Markov assumption [Eq. (23)] implicitly assumed for $t=2,3$, and 4 in drawing Fig. 3 roughly holds true at least up to $t \approx 4$ for large $n$.

Theoretically, most sequences $Y_{t}$ that meet $X_{t}$ after $t$ steps of state transition own the sequence of distance given by $\boldsymbol{d}_{t}^{*}=$ $\left\{d_{0}^{*}, d_{1}^{*}, \ldots, d_{t-1}^{*}, d_{t}^{*}=0\right\}$. In particular, a majority of the initial states $Y_{0}$ is initially separated from $X_{0}$ by $d_{0}^{*}(t)$. Figure 4 suggests that this is the case at least up to $t \approx 4$ for large $n$. The sequence of distances $\boldsymbol{d}_{t}^{*}$ is shown for $1 \leqslant t \leqslant 4$ in Fig. 5.

\section{IN-DEGREE DISTRIBUTION OF THE STATE TRANSITION GRAPH}

We calculate the incoming degree distribution

$$
p_{k}=\operatorname{Prob}\left\{\left|f^{-1} \boldsymbol{x}\right|=k\right\},
$$

where $k$ is the in-degree of a state and $\sum_{k=0}^{N} p_{k}=1$. Because each node in the state transition graph has exactly one outgoing

TABLE I. Theoretical estimates of $c_{t}=\ln \bar{S}_{t} / n$.

\begin{tabular}{lccc}
\hline \hline$t$ & SBNs & DMNs & SMNs \\
\hline 1 & 0.223 & 0.157 & 0.256 \\
2 & 0.323 & 0.205 & 0.363 \\
3 & 0.380 & 0.225 & 0.421 \\
4 & 0.416 & 0.232 & 0.460 \\
\hline \hline
\end{tabular}




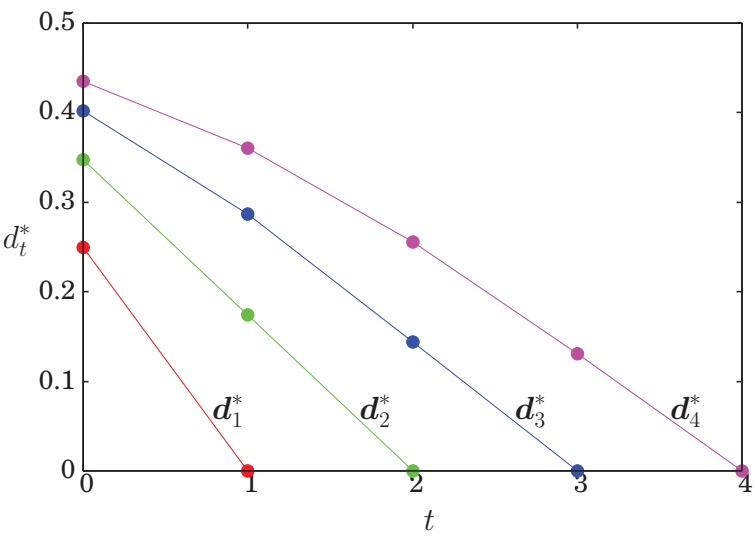

FIG. 5. (Color online) Sequence of the most likely distances $\boldsymbol{d}_{t}^{*}=$ $\left\{d_{0}^{*}, d_{1}^{*}, \ldots, d_{t-1}^{*}, d_{t}^{*}=0\right\}$ for $1 \leqslant t \leqslant 4$.

link, we have

$$
\langle k\rangle=\sum_{k=0}^{N} k p_{k}=1
$$

where $\langle\cdot\rangle$ indicates the expectation. Because $r_{k}=k p_{k}[8]$ (also see [21] for an example), we obtain

$$
\left\langle k^{2}\right\rangle=\sum_{k=0}^{N} k^{2} p_{k}=\sum_{k=0}^{N} k r_{k}=\bar{S}_{1}=e^{n c_{1}} .
$$

Therefore, $c_{1}>0$ indicates that $\left\langle k^{2}\right\rangle$ diverges in the limit of $N=2^{n} \rightarrow \infty$, reminiscent of the scale-free property of the state transition graph [21-23].

For DBNs, the state transition graph is the directed random graph in which $p_{k}$ obeys the Poisson distribution (i.e., $p_{k}=$ $1 / e k$ !) with mean and variance $1[5,6]$. Therefore, $\left\langle k^{2}\right\rangle=2$, proving that $c_{1}=0$ for DBNs (i.e., there is no exponential state concentration). This is consistent with Fig. 4(a) (circles). Figure 3 suggests that $c_{1} \approx 0$ when $K$ is larger than $\cong 10$. Therefore, the in-degree distribution of the state transition graph is also narrow for SBNs with $K \geqslant 10$. We verified that the numerically obtained in-degree distribution for the random Boolean network with $n=30$ and $K=20$ approximately obeys the Poisson distribution [Fig. 6(a)].

In contrast, the positive value of $c_{1}$ found for SBNs with small $K$, DMNs, and SMNs (Fig. 3) indicates that $\left\langle k^{2}\right\rangle\left(=\bar{S}_{1}\right)$ diverges exponentially in $n$. This is actually the case, as shown in Figs. 4(b)-4(d). For scale-free networks with $p_{k} \approx k^{-\gamma}$, the extremal criterion would lead to $\gamma \approx\left(c_{1}+3 \ln 2\right) /\left(c_{1}+\right.$ $\ln 2)[22,23]$. However, the in-degree distribution numerically obtained for majority vote networks, shown in Figs. 6(c) and 6(d), deviates from a power law. The in-degree distribution numerically obtained for SBNs [Fig. 6(b)] is also different from a power law [20]. To guide the eyes, fitting curves on the basis of a power law with an exponential cutoff are shown by the lines in Figs. 6(b)-6(d). In fact, the power law is not the only distribution that yields the divergence of $\left\langle k^{2}\right\rangle$. In the present case, the position of the exponential cutoff may mildly diverge as $n$ becomes large.
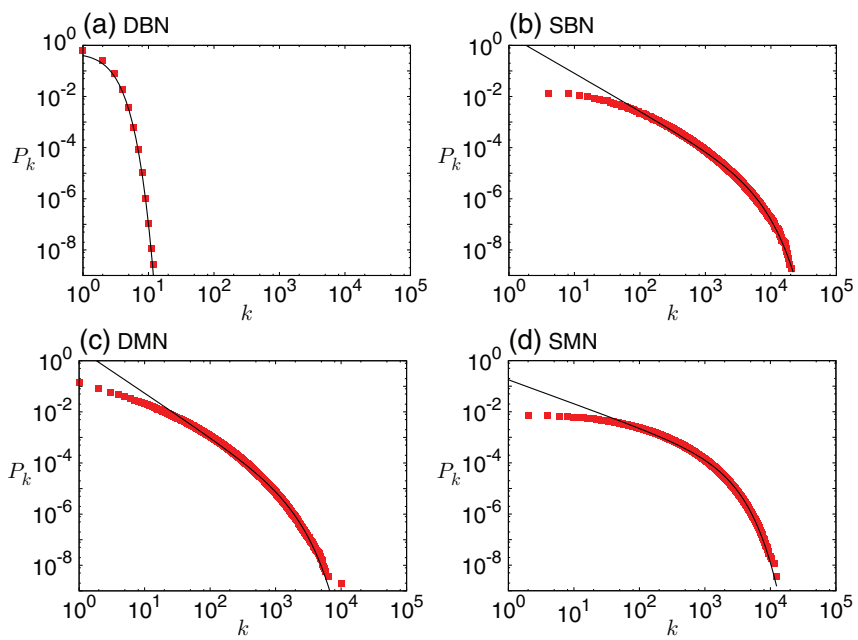

FIG. 6. (Color online) Complementary in-degree distribution of $p_{k}$ (i.e., $P_{k} \equiv \sum_{k^{\prime}=k}^{N} p_{k^{\prime}}$ ) of the state transition graph. (a) DBNs, ( $n=30$ and $K=30$ ). The numerical results are shown by squares, and the Poisson distribution with mean 1 is indicated by the line. (b) SBNs, $(n=30$ and $K=3)$. (c) DMNs, $(n=30$ and $K=30)$. (d) SMNs, $(n=30$ and $K=3)$. The fitting curves, $P_{k} \propto k^{-1.48} \exp \{-0.000298 k\}, P_{k} \propto k^{-1.72} \exp \{-0.000985 k\}$, and $P_{k} \propto k^{-0.936} \exp \{-0.000786 k\}$, for (b), (c), and (d), respectively, obtained from the least square error method, are shown by the lines as guides to the eyes.

\section{DISCUSSION AND CONCLUSIONS}

In summary, we provided a unified framework for analyzing the state concentration. We found that exponential state concentration occurs in SBNs with small $K$ and the majority vote networks with arbitrary $K$ (i.e., DMNs and SMNs), but not in DBNs. We also revealed the long-tailed distributions of the in-degree of the state transition graph in SBNs, DMNs, and SMNs, but not in DBNs.

We briefly discuss the relationship between the quickness, measured by the exponent of the state concentration in this study, and the robustness of the dynamics. The robustness of the dynamics is often measured in terms of damage spreading. It is a long-term property concerning the stability of $d=0$ for mapping $\varphi$. As we mentioned, $d=0$ is an unstable fixed point of $\varphi$ unless $K=1$ or 2 . Although SMNs with $K=1$ or 2 satisfies quickness and robustness, we do not discuss these cases because the dynamics in these cases is just frozen $[18,19]$. Here we consider a simpler measure of robustness based on a one-step property, i.e., how a difference by a single bit evolves after a single application of $f$. This is essentially the same as the Boolean derivative, a measure of the robustness used for analyzing random Boolean networks [12-15]. In SMNs, the probability that a single bit flip in input results in a bit flip after the application of $f$ is given by

$$
g_{K, 1}=(2 / \pi) \sin ^{-1} \sqrt{1 / K} .
$$

Because $g_{K, 1}$ is equal to 0.5 for $K=2$ and decreases according to $\approx 2 /(\pi \sqrt{K})$ as $K$ increases, the random majority vote network is robust except for very small $K$. However, in the random Boolean network, the same flip probability is equal to 
$1 / 2-1 / 2^{2^{K}}$, which quickly approaches $1 / 2$ as $K$ increases. In particular, the flip probability for the Boolean network at $K=2$ is equal to $7 / 16=0.4375$, which is close to the values for the majority vote network with $K=2$ (i.e., 0.5 ) and $K=3$ (i.e., 0.392). Although the Boolean network with $K=1$ has sufficient robustness, the dynamics in this case is frozen [24]. Therefore, we consider that the robustness of the one-step dynamics holds true in DMNs, but not in DBNs, SBNs, and SMNs. By combining this observation with our main results, we conclude that quickness and robustness are suitably balanced in DMNs, but not in DBNs, SBNs, and SMNs.
[1] S.-I. Amari, IEEE Trans. Comput. 11, 1197 (1972).

[2] J. J. Hopfield, Proc. Natl. Acad. Sci. USA 79, 2554 (1982).

[3] J. Hertz, A. Krogh, and R. G. Palmer, Introduction to the Theory of Neural Computation (Westview Press, Boulder, CO, 1991).

[4] S. A. Kauffman, J. Theor. Biol. 22, 437 (1969).

[5] S. A. Kauffman, Physica D 10, 145 (1984).

[6] S. A. Kauffman, The Origin of Orders: Self-Organization and Selection in Evolution (Oxford University Press, Oxford, 1993).

[7] S. Bornholdt, J. R. Soc. Interface 5, 85 (2008).

[8] S. Amari, Kybernetik 14, 201 (1974).

[9] S. Amari, K. Yoshida, and K. Kanatani, SIAM J. Appl. Math. 33, 95 (1977).

[10] S. Amari, Proc. IEEE 78, 1443 (1990).

[11] C. Castellano, S. Fortunato, and V. Loreto, Rev. Mod. Phys. 81, 591 (2009).

[12] B. Luque and R. V. Solé, Phys. Rev. E 55, 257 (1997).

[13] B. Luque and R. V. Solé, Physica A 284, 33 (2000).
[14] S. Kauffman, C. Peterson, B. Samuelsson, and C. Troein, Proc. Natl. Acad. Sci. USA 101, 17102 (2004).

[15] I. Shmulevich and S. A. Kauffman, Phys. Rev. Lett. 93, 048701 (2004).

[16] A. Bhattacharjya and S. Liang, Phys. Rev. Lett. 77, 1644 (1996).

[17] B. Derrida and Y. Pomeau, Europhys. Lett. 1, 45 (1986); B. Derrida and G. Weisbuch, J. Phys. (Paris) 47, 1297 (1986).

[18] B. Derrida, J. Phys. A 20, L721 (1987).

[19] K. E. Kürten, Phys. Lett. A 129, 157 (1988); J. Phys. A 21, L615 (1988).

[20] A. Shreim, A. Berdahl, V. Sood, P. Grassberger, and M. Paczuski, New J. Phys. 10, 013028 (2008).

[21] M. E. J. Newman, S. H. Strogatz, and D. J. Watts, Phys. Rev. E 64, 026118 (2001).

[22] M. E. J. Newman, Contemp. Phys. 46, 323 (2005).

[23] S. N. Dorogovtsev, A. V. Goltsev, and J. F. F. Mendes, Rev. Mod. Phys. 80, 1275 (2008).

[24] H. Flyvbjerg and N. J. Kjaer, J. Phys. A 21, 1695 (1988). 